\title{
Constraints on dark matter annihilation from AMS-02 results
}

\author{
Joachim Kopr* ${ }^{*}$ \\ Max Planck Institut für Kernphysik, \\ Saupfercheckweg 1, 69117 Heidelberg, Germany
}

(Dated: August 15, 2018)

\begin{abstract}
We use recently released data on the positron-to-electron ratio in cosmic rays from the AMS-02 experiment to constrain dark matter annihilation in the Milky Way. Due to the yet unexplained positron excess, limits are generally weaker than those obtained using other probes, especially gamma rays. This also means that explaining the positron excess in terms of dark matter annihilation is difficult. Only if very conservative assumptions on the dark matter distribution in the Galactic Center region are adopted, it may be possible to accommodate dark matter annihilating to leptons with a cross section above $10^{-24} \mathrm{~cm}^{3} / \mathrm{sec}$. We comment on several theoretical mechanisms to explain such large annihilation cross sections.
\end{abstract}

PACS numbers: 95.35.+d, 96.50.sb

Recently, the AMS-02 collaboration has announced a new measurement of the cosmic ray positron fraction, i.e. the flux of positrons, divided by the flux of electrons plus positrons [1. AMS-02 confirms an increase in the positron fraction at energies above $\sim 10 \mathrm{GeV}$, which had been observed previously by PAMELA 2 2 and FermiLAT [3. This upturn is difficult to explain by secondary production of positrons in interactions of other high energy cosmic rays, but suggests the existence of a yet unidentified galactic source of positrons [4. One interesting candidate are pulsars, fast rotating neutron stars in whose strong electromagnetic field high energy $e^{+} e^{-}$ pairs are produced (see ref. 4] and references therein for details).

Another possible source of primary positrons is dark matter (DM) annihilation or decay into high energy Standard Model particles. This possibility has been extensively discussed in the context of the PAMELA and Fermi-LAT results [2, 3, see for instance [5] 17]. The conclusion is that explaining the positron excess in terms of dark matter is very difficult: the required annihilation cross sections are much larger than the ones required to explain the DM abundance in the Universe through thermal freeze-out. (Several mechanisms have been proposed to make the annihilation cross section velocity dependent and thus different in the early Universe and today [6, 9, Also, one expects the production of high energy particles to be accompanied by radio signals from synchrotron radiation and by gamma rays from decays of unstable annihilation products (in particular $\pi^{0} \rightarrow \gamma \gamma$ ), from final state radiation and from inverse Compton scattering. While the magnitude of these signals relative to the positron signal depends strongly on poorly understood details of galactic modeling (DM profile, magnetic fields, etc.), particle physics models capable of explaining the positron excess are often in tension with constraints from gamma ray and radio observations [8, 18, 19].

In this note, we discuss dark matter annihilation in view of the new AMS-02 measurements and derive constraints on the DM mass and annihilation cross section.

Methods. We take the primary spectrum of positrons from DM annihilation from [20, 21. For the fluxes after propagation we use the results from [20, as well results we have obtained with GALPROP v54 22, 26], which we have modified to allow for the inclusion of arbitrary injection spectra. We have checked that, for identical propagation models, we can reproduce the results from 20 in GALPROP to within few $\times 10 \%$. We consider the annihilation channels $\chi \chi \rightarrow \mu^{+} \mu^{-}, \chi \chi \rightarrow \tau^{+} \tau^{-}$, $\chi \chi \rightarrow b \bar{b}, \chi \chi \rightarrow W^{+} W^{-}$and $\chi \chi \rightarrow Z Z$, where $\chi$ is the DM particle. To illustrate the impact of uncertainties in charged cosmic ray propagation through the galaxy, we will show limits for the three different propagation models MIN, MED and MAX from [20], originally introduced in [27, 28]. To derive limits on the DM annihilation cross sections, we compare the theoretically predicted positron flux to the data from [1. We use only data points above $20 \mathrm{GeV}$ to be insensitive to modulation by varying solar activity. We extract the positron flux $\phi\left(e^{+}\right)$from the observed positron fraction $\phi\left(e^{+}\right) /\left[\phi\left(e^{+}\right)+\phi\left(e^{-}\right)\right]$by multiplying the latter with the total electron plus positron flux $\phi\left(e^{+}\right)+\phi\left(e^{-}\right)$measured by Fermi-LAT [29]. To avoid any reliance on theoretical modelling of the background from secondary positrons, we set conservative limits by requiring that the predicted positron flux remains smaller than the measured one (within error bars) at all energies. More precisely, we define

$$
\chi^{2}=\sum_{i} \frac{\left(\phi_{e^{+}, i}^{\mathrm{th}}-\phi_{e^{+}, i}^{\mathrm{obs}}\right)^{2}}{\sigma_{i}^{2}} \theta\left(\phi_{e^{+}, i}^{\mathrm{th}}-\phi_{e^{+}, i}^{\mathrm{obs}}\right),
$$


where $\phi_{e^{+}, i}^{\text {th }}$ and $\phi_{e^{+}, i}^{\text {obs }}$ denote the predicted and observed fluxes, respectively, $\sigma_{i}$ are the experimental errors, and the sum runs over energy bins. We set a one-sided $95 \%$ C.L. limit by requiring $\chi^{2}<4.6$. The relative uncertainties $\sigma_{i} / \phi_{e+, i}^{\text {obs }}$ are conservatively obtained by adding linearly the uncertainties of the AMS-02 positron fraction and of the Fermi-LAT $e^{+}+e^{-}$flux. Within each experimental data set, we have added the quoted statistical and systematic uncertainties in quadrature.

We will also explore the possibility that the positron excess is explained by DM annihilation. This requires some assumptions on the background flux of secondary astrophysical positrons. Here, we use GALPROP v54 22 26] for both the signal and the background, with input parameters tuned to reproduce Fermi's $e^{+}+e^{-}$spectrum 29] at energies between few $\mathrm{GeV}$ and few $\times 10 \mathrm{GeV}$, i.e. in an energy range where the $e^{+}+e^{-}$flux from annihilation of heavy DM is expected to be negligible. Our parameter choice is motivated by references [29, 30]. To quantify the uncertainties in the propagation model, we also show results obtained using GALPROP implementations of the MIN, MED and MAX models from [20, 27, 28 discussed above. To obtain the preferred parameter regions, we define in analogy to equation (1)

$$
\chi^{2}=\sum_{i} \frac{\left(\phi_{e^{+}, i}^{\mathrm{th}}-\phi_{e^{+}, i}^{\mathrm{obs}}\right)^{2}}{\sigma_{i}^{2}},
$$

where $\phi_{e^{+}, i}^{\text {th }}$ now includes the signal and background predictions. We set two-sided " $3 \sigma$ " CL limits by requiring $\chi^{2}<11.8$. The quotation marks here indicate that the confidence level is based on statistical errors only because the probability distributions of the systematic biases in the propagation and backgrounds model are unknown.

Results and discussion. In figure 1 we show the limits on the DM annihilation cross section $\left\langle\sigma v_{\text {rel }}\right\rangle$ and the DM mass $m_{\chi}$ derived from AMS-02 data. ( $\left\langle\sigma v_{\text {rel }}\right\rangle$ denotes the annihilation cross section, multiplied by the relative velocity of the two annihilating DM particles and averaged over their velocity distribution.) Solid black lines correspond to the MED propagation model, whereas red bands indicate the difference between the MIN and MAX propagation models. We have assumed the DM distribution $\rho(r)$ in the Milky Way to follow the NFW profile [36] $\rho_{\mathrm{NFW}}=\rho_{s}\left(r_{s} / r\right)\left(1+r / r_{s}\right)^{-2}$ with the parameters $\rho_{s}=0.184 \mathrm{GeV} / \mathrm{cm}^{3}, r_{s}=24.42 \mathrm{kpc}$ [20, but we have verified that the choice of halo profile has a negligible impact on our limits. The reason is that high energy positrons cannot travel too far in the Milky Way before losing energy, so that the flux observed at the Earth has to come from our local galactic neighborhood, where the DM halo profile has relatively small uncertainties. We compare the AMS-02 limits to the bounds obtained by Cirelli and Giesen from the antiproton fluxes measured in PAMELA [33, 34, to the bounds obtained by the Fermi-LAT collaboration from an analysis of gamma ray signals from dwarf galaxies [31] (see also [37]), to the bounds obtained by Hooper et al. using Fermi-LAT gamma ray observations of the Galactic Center 32] (see also [8, 19, 38]), and to limits from inverse Compton scattering (ICS) [18, again based on Fermi-LAT data. Note that the antiproton constraints are based on a particular cosmic ray propagation model and can vary by more than an order of magnitude if the propagation parameters are changed. Gamma ray signals from the Galactic Center on the other hand depend very strongly on the details of the DM distribution in that region. We use here the most conservative limits from [32, based on the assumption of a cored profile. Significantly stronger limits are obtained for steeper profiles, which are also in better agreement with simulations of galaxy formation and evolution (see 32 for details). For the ICS limits, we depict the uncertainty in the DM density distribution as yellow bands. The limits are taken from 18] and are based on Fermi data from a $3^{\circ} \times 3^{\circ}$ region around the Galactic Center. ICS exclusion limits derived from larger regions of interest are less dependent on the DM density distribution and would lie close to the upper ends of the yellow bands in fig. 1. Note that IceCube limits on neutrinos from DM annihilation [39] are still outside the parameter range shown in fig. 1, but can be expected to improve with more statistics.

We see that for most annihilation channels, AMS-02 constraints are weaker than bounds from PAMELA and Fermi-LAT. Only for annihilation to $\mu^{+} \mu^{-}$, AMS-02 and Fermi-LAT $\gamma$ ray limits can be comparable, but only when very conservative assumptions on the DM halo profile are made to avoid ICS bounds. The reason why hadronic annihilation channels are more strongly constrained by Fermi-LAT data is the occurrence of prompt $\gamma$ rays from $\pi^{0}$ decay. Antiproton constraints are not competitive in the $\mu^{+} \mu^{-}$and $\tau^{+} \tau^{-}$channels and are therefore not included in figure 1 for these channels. Fermi-LAT results are not available for the $Z Z$ final state, but are expected to be similar to those for the $W^{+} W^{-}$final state 8 .

For the $\mu^{+} \mu^{-}$and $\tau^{+} \tau^{-}$final states, we also illustrate in figure 1 the values of $m_{\chi}$ and $\left\langle\sigma v_{\text {rel }}\right\rangle$ that would be needed to explain the positron excess in terms of dark matter (green shaded regions). For the $b \bar{b}, W^{+} W^{-}$and $Z Z$ final states, we find that the predicted positron spectrum is typically too flat to explain the data. This is also illustrated in figure 2, where we compare several specific DM scenarios to AMS-02 data. We conclude from figure 1 that interpreting the observed $e^{+}$excess in terms of dark matter remains difficult. On the one hand, constraints from antiproton and gamma ray observations are extremely constraining, especially for the $b \bar{b}, W^{+} W^{-}$and $Z Z$ final states. We emphasize again that the Galactic Center gamma ray limits shown in figure 1 are very conservative, and significantly stronger limits are obtained 

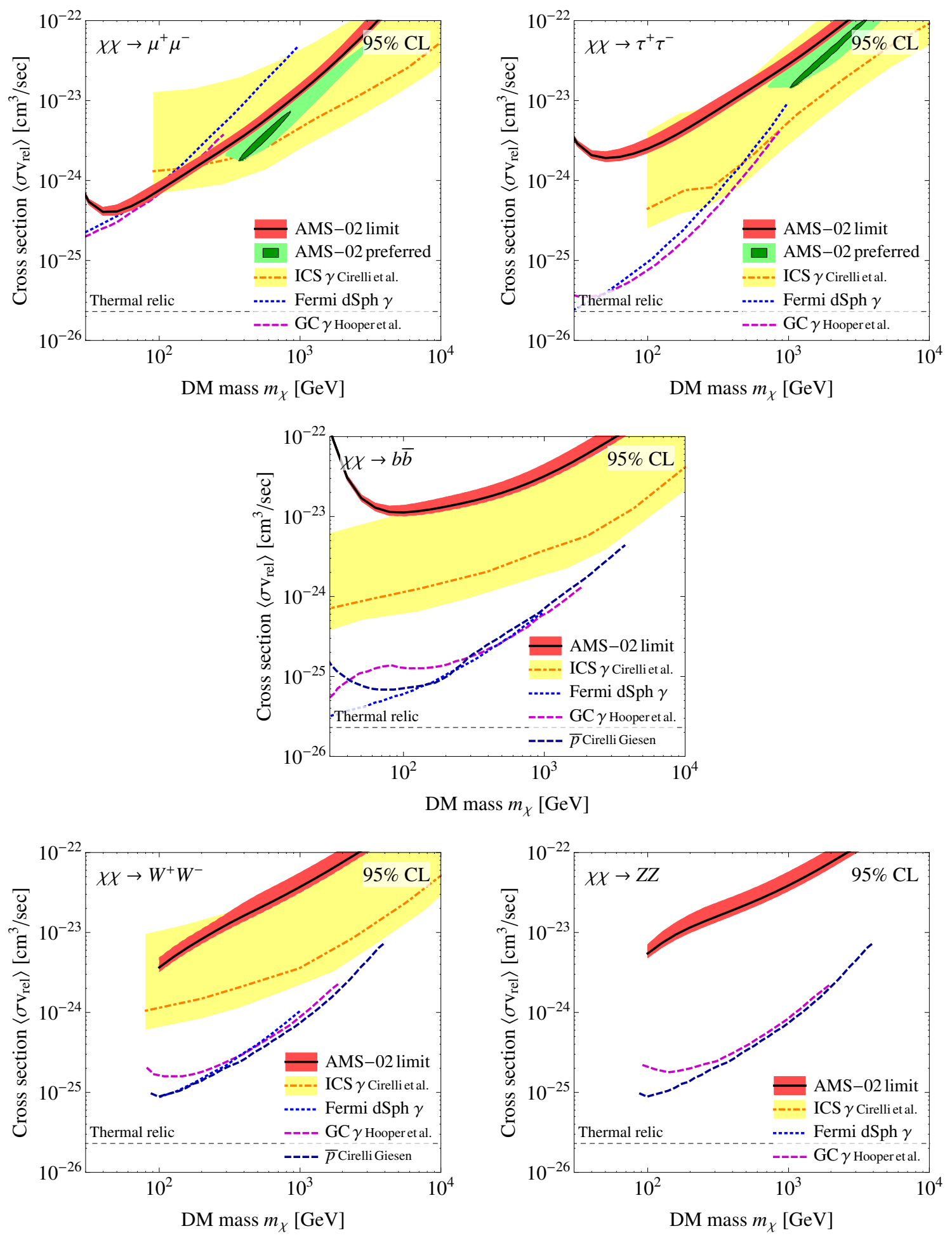

Figure 1: Limits on the DM annihilation cross section $\left\langle\sigma v_{\text {rel }}\right\rangle$ as a function of the DM mass $m_{\chi}$ for the annihilation channels $\chi \chi \rightarrow \mu^{+} \mu^{-}$(top left), $\chi \chi \rightarrow \tau^{+} \tau^{-}$(top right), $\chi \chi \rightarrow b \bar{b}$ (middle), $\chi \chi \rightarrow W^{+} W^{-}$(bottom left), $\chi \chi \rightarrow Z Z$ (bottom right). Solid black lines show constraints derived in this work from AMS-02 positron data, and red bands indicate how uncertainties in the positron propagation model 20 affect these constraints. We have assumed an NFW profile for the DM distribution in the Milky Way, but have checked that alternative choices lead to almost identical limits. Where available, we show also for comparison limits from Fermi-LAT observations of $\gamma$ ray emission in dwarf galaxies [31 (light blue dotted) and in the Galactic Center 32 (purple dashed), from an analysis of PAMELA antiproton data 33 34 (dark blue dashed), and from inverse Compton scattering [18] (orange line $=$ NFW profile, lower (upper) edge of yellow band = Einasto (isothermal) profile). The horizontal dashed line shows the annihilation cross section that yields the correct DM abundance via freeze-out [35. For $\chi \chi \rightarrow \mu^{+} \mu^{-}$and $\chi \chi \rightarrow \tau^{+} \tau^{-}$we indicate in green the parameter regions that would be favored by attempts to explain the positron excess in terms of DM annihilation (dark green = background and propagation models based on Fermi observations, light green $=$ MIN, MED, MAX propagation models). 


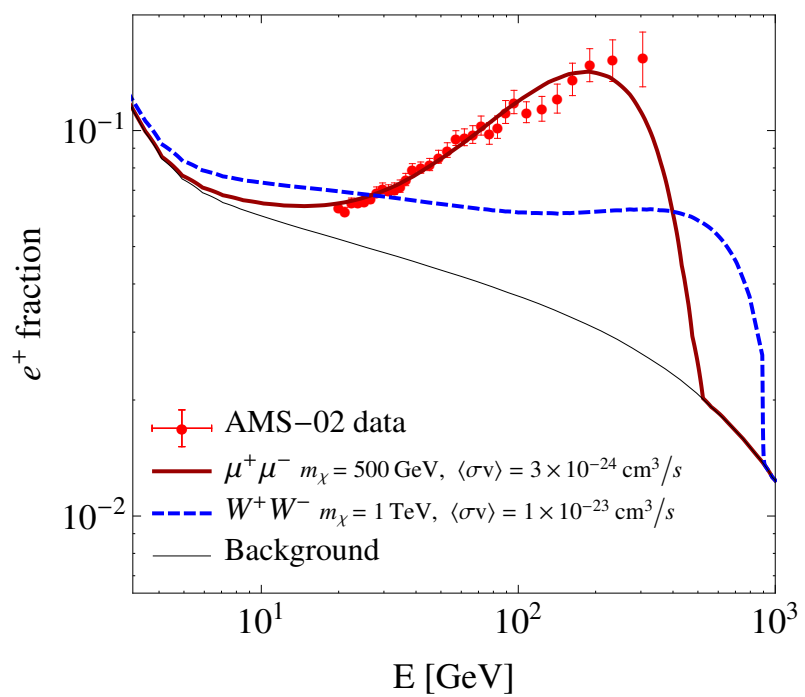

Figure 2: Comparison of the positron fraction measured by AMS-02 to two particular dark matter scenarios.

for less conservative assumptions on the dark matter profile in the Galactic Center region. Even if the constraints are avoided, the annihilation cross section required to explain the positron data is significantly larger than the thermal relic value $\left\langle\sigma v_{\text {rel }}\right\rangle \simeq 2.3 \times 10^{-26} \mathrm{~cm}^{3} / \mathrm{sec} 35$. (horizontal dashed line in figure 1). Mechanisms to enhance $\left\langle\sigma v_{\text {rel }}\right\rangle$ today while retaining a smaller value in the early Universe include Sommerfeld enhancement [6, 40] and resonant enhancement 9. In both of these scenarios, $\left\langle\sigma v_{\text {rel }}\right\rangle$ acquires a velocity dependence, which leads to small cross sections at the time of DM freeze-out $\left(\left\langle v_{\text {rel }}^{2}\right\rangle \simeq 0.24 c^{2}\right)$, but larger cross sections in the Milky Way today $\left(v_{\text {rel }} \sim\right.$ few $\left.\times 100 \mathrm{~km} / \mathrm{sec}\right)$. In dwarf galaxies, DM velocities are even smaller, of order few $\times 10 \mathrm{~km} / \mathrm{sec}$. Fermi-LAT gamma ray limits from dwarf galaxies will impose severe constraints on such models because of the small velocity dispersion in dwarf galaxies. Constraints are typically weakest for final states composed exclusively of light charged leptons: antiprotons are not produced in this case, and the expected gamma ray flux is significantly weaker than for final states containing hadrons. An interesting possibility in this context is DM annihilating to a light intermediate state which then decays into leptons [11, with decays to heavier particles forbidden by the low mass of the intermediate state. Other possibilities are that DM is non-thermally produced in the early Universe (for instance through decays of a long-lived intermediate state), or that there are several dark matter components: a dominant one that drives galactic dynamics, and a subdominant one that exhibits stronger clustering (for instance due to relatively large self-interactions), leading to locally larger densities (see for instance [4]).
Conclusions. We have derived limits on dark matter annihilation from the cosmic ray positron fraction measured by AMS-02. Due to the yet unexplained positron excess, these limits are in most cases not competitive with constraints from gamma ray and antiproton observations. On the other hand, models capable of explaining the positron excess through DM annihilation are severely constrained experimentally by the non-observation of anomalous gamma ray and antiproton populations, and theoretically by the requirement of extremely large annihilation cross sections. Fitting AMS-02 data in such models may be marginally possible if the annihilation is exclusively to leptons, and if very conservative assumptions on the DM profile in the Galactic Center region are adopted to weaken $\gamma$ ray constraints.

Note added: After the first version of this manuscript appeared on the arXiv, several studies have found possible tension between the AMS-02 positron fraction and Fermi-LAT data on the $e^{+}+e^{-}$spectrum, see for instance [42 44]. In particular, it is difficult to explain both data sets simultaneously with a new population of highenergy electrons and positrons (coming e.g. from DM annihilation or decay or from an astrophysical source), as long as this population is charge-symmetric. It is at the moment unclear whether the tension is due to a problem with the propagation model for electrons and positrons, due to an underestimated systematic effect in one of the experiments, or due to a charge asymmetry in the new $e^{+} e^{-}$population [44, 45]. If future studies should reveal that the tension is due to experimental systematics, also the uncertainties in our procedure of extracting the positron flux by combining AMS-02 and Fermi data would increase.

Acknowledgment I would like to thank CERN for kind hospitality and support during part of this work.

* Email: jkopp@mpi-hd.mpg.de

[1] The AMS-02 collaboration (2013), talk given by S. Ting at Cern on Apr 03, 2013; main results available from http://www.ams02.org/.

[2] O. Adriani et al. (PAMELA Collaboration), Nature 458, 607 (2009), 0810.4995.

[3] M. Ackermann et al. (Fermi LAT Collaboration), Phys.Rev.Lett. 108, 011103 (2012), 1109.0521.

[4] P. D. Serpico, Astropart.Phys. 39-40, 2 (2012), 1108.4827.

[5] M. Cirelli, M. Kadastik, M. Raidal, and A. Strumia, Nucl.Phys. B813, 1 (2009), 0809.2409.

[6] N. Arkani-Hamed, D. P. Finkbeiner, T. R. Slatyer, and N. Weiner, Phys.Rev. D79, 015014 (2009), 0810.0713.

[7] E. Nardi, F. Sannino, and A. Strumia, JCAP 0901, 043 (2009), 0811.4153.

[8] G. Bertone, M. Cirelli, A. Strumia, and M. Taoso, JCAP 0903, 009 (2009), 0811.3744.

[9] M. Ibe, H. Murayama, and T. Yanagida, Phys.Rev. D79, 
095009 (2009), 0812.0072.

[10] P. Meade, M. Papucci, and T. Volansky, JHEP 0912, 052 (2009), 0901.2925.

[11] J. Mardon, Y. Nomura, D. Stolarski, and J. Thaler, JCAP 0905, 016 (2009), 0901.2926.

[12] V. Barger, Y. Gao, W. Y. Keung, D. Marfatia, and G. Shaughnessy, Phys.Lett. B678, 283 (2009), 0904.2001.

[13] P. Meade, M. Papucci, A. Strumia, and T. Volansky, Nucl.Phys. B831, 178 (2010), 0905.0480.

[14] S. Profumo and T. E. Jeltema, JCAP 0907, 020 (2009), 0906.0001.

[15] M. Cirelli, F. Iocco, and P. Panci, JCAP 0910, 009 (2009), 0907.0719.

[16] J. M. Cline, A. C. Vincent, and W. Xue, Phys.Rev. D81, 083512 (2010), 1001.5399.

[17] A. Calvez, W. Essey, M. Fairbairn, A. Kusenko, and M. Loewenstein, Astropart.Phys. 35, 185 (2011), 1003.1113.

[18] M. Cirelli, P. Panci, and P. D. Serpico, Nucl.Phys. B840, 284 (2010), 0912.0663.

[19] R. Crocker, N. Bell, C. Balazs, and D. Jones, Phys.Rev. D81, 063516 (2010), 1002.0229.

[20] M. Cirelli, G. Corcella, A. Hektor, G. Hutsi, M. Kadastik, et al., JCAP 1103, 051 (2011), 1012.4515.

[21] P. Ciafaloni, D. Comelli, A. Riotto, F. Sala, A. Strumia, et al., JCAP 1103, 019 (2011), 1009.0224.

[22] I. Moskalenko and A. Strong, Astrophys.J. 493, 694 (1998), astro-ph/9710124.

[23] A. Strong and I. Moskalenko, Astrophys.J. 509, 212 (1998), astro-ph/9807150.

[24] V. Ptuskin, I. V. Moskalenko, F. Jones, A. Strong, and V. Zirakashvili, Astrophys.J. 642, 902 (2006), astro$\mathrm{ph} / 0510335$.

[25] A. W. Strong, I. V. Moskalenko, and V. S. Ptuskin, Ann.Rev.Nucl.Part.Sci. 57, 285 (2007), astro$\mathrm{ph} / 0701517$.

[26] A. E. Vladimirov, S. W. Digel, G. Johannesson, P. F. Michelson, I. V. Moskalenko, et al., Comput.Phys.Commun. 182, 1156 (2011), http://galprop.stanford.edu/webrun/, 1008.3642.

[27] T. Delahaye, R. Lineros, F. Donato, N. Fornengo, and P. Salati, Phys.Rev. D77, 063527 (2008), 0712.2312.

[28] F. Donato, N. Fornengo, D. Maurin, and P. Salati, Phys.Rev. D69, 063501 (2004), astro-ph/0306207.

[29] M. Ackermann et al. (Fermi LAT Collaboration), Phys.Rev. D82, 092004 (2010), 1008.3999.

[30] A. W. Strong (2009), http://www.mpe.mpg.de/ aws/propagate.html see in particular file galdef_54_77Xvarh7S.

[31] M. Ackermann et al. (Fermi-LAT collaboration), Phys.Rev.Lett. 107, 241302 (2011), 1108.3546.

[32] D. Hooper, C. Kelso, and F. S. Queiroz (2012), 1209.3015.

[33] O. Adriani et al. (PAMELA Collaboration) (2010), arXiv:1007.0821.

[34] M. Cirelli and G. Giesen (2013), 1301.7079.

[35] G. Steigman, B. Dasgupta, and J. F. Beacom (2012), 1204.3622 .

[36] J. F. Navarro, C. S. Frenk, and S. D. M. White, Astrophys. J. 462, 563 (1996), astro-ph/9508025.

[37] A. Geringer-Sameth and S. M. Koushiappas, Phys.Rev.Lett. 107, 241303 (2011), 1108.2914.

[38] M. Regis and P. Ullio, Phys.Rev. D78, 043505 (2008), 0802.0234 .

[39] R. Abbasi et al. (IceCube Collaboration), Phys.Rev. D84, 022004 (2011), 1101.3349.

[40] J. Hisano, S. Matsumoto, M. Nagai, O. Saito, and M. Senami, Phys.Lett. B646, 34 (2007), hep$\mathrm{ph} / 0610249$.

[41] J. Fan, A. Katz, L. Randall, and M. Reece (2013), 1303.1521.

[42] I. Cholis and D. Hooper (2013), 1304.1840.

[43] Q. Yuan, X.-J. Bi, G.-M. Chen, Y.-Q. Guo, S.-J. Lin, et al. (2013), 1304.1482.

[44] L. Feng and Z. Kang (2013), 1304.7492.

[45] I. Masina and F. Sannino, JCAP 1109, 021 (2011), 1106.3353. 